\title{
Linx
}

Revue des linguistes de l'université Paris X Nanterre

$55 \mid 2006$

Thème et thématisation

\section{L'entrée thème/rhème du glossaire de Comenius}

\section{Danièle Flament}

\section{OpenEdition \\ Journals}

Édition électronique

URL : http://journals.openedition.org/linx/389

DOI : $10.4000 / \operatorname{lin} x .389$

ISSN : 2118-9692

\section{Éditeur}

Presses universitaires de Paris Nanterre

\section{Édition imprimée}

Date de publication : 1 décembre 2006

Pagination : 61-71

ISSN : 0246-8743

\section{Référence électronique}

Danièle Flament, «L'entrée thème/rhème du glossaire de Comenius », Linx [En ligne], 55 | 2006, mis en ligne le 22 février 2011, consulté le 19 avril 2019. URL : http://journals.openedition.org/linx/389 ; DOI 10.4000/linx.389 


\title{
L'entrée thème/rbème du glossaire de Comenius
}

\author{
Danièle Flament \\ UMR 7114 Paris X/CNRS Modyco \\ Daniele.Boistrancourt@kuleuven-kortrijk.be \\ Avec la collaboration de François Muller et d'Anne Trévise
}

Entrée thème/rhème : «L'opposition thème/rhème est une opposition de nature informationnelle, qui vise à distinguer dans l'énoncé, d'une part le support de l'information (le thème), d'autre part l'information qui est communiquée à propos de ce support (le rhème)» (Neveu, 2000, p. 113). Ceci n'implique pas pour autant que tout énoncé se distribue en un thème et un rhème. Les mots thème et rhème viennent du grec thema (ce qui est posé) et rhema (mot, parole). Il existe à côté du couple thème/rhème de nombreuses autres oppositions voisines: thème/propos (Bally, 1932), thème/ commentaire, topique/ commentaire (Hockett, 1958), thème/focus (Sgall, 1973), donné/nouveau (Halliday, 1967), préfixe/noyau (Blanche-Benveniste, 1997). Comme l'écrit Galmiche (1992, p. 4), «cette prolifération [dans les dénominations] est liée au fait que le phénomène en question ressortit à une multiplicité de points de vue: sémantique, logique, psychologique, pragmatique, énonciatif, à quoi se mêlent des considérations relatives à la phonologie (intonation) et à la syntaxe (constructions) ». Cette multiplicité n'a pas manqué d'entraîner des confusions. Ainsi, le mot focus, souvent employé pour désigner le rhème, le foyer d'information, peut désigner chez certains auteurs le thème.

CF : sujet/prédicat, cohésion (anaphore, cataphore), focalisation, postrhème, ordre des mots, texte. 
Histoire: Dès le XIX ${ }^{\mathrm{e}}$ siècle, certains linguistes allemands établissent une distinction entre le "psychologisches Subjekt» et le "psychologisches Objekt» d'un discours (voir par exemple Paul, 1880 et von der Gabelentz, 1891). C'est cependant le linguiste Charles Bally (1932) qui est habituellement considéré comme le premier à avoir proposé l'opposition bien connue thème/propos: «la pensée qu'on veut faire connaître est - nous le répétons - le but, la fin de l'énoncé, ce qu'on propose, en un mot: le propos; on l'énonce à l'occasion d'une autre chose qui en forme la base, le substrat, le motif : c'est le thème» (rééd. 1965, p. 52). On notera que cette distinction apparaît aussi dans les années 30 chez les linguistes du Cercle de Prague, qui développent alors une " perspective fonctionnelle de la phrase ».

\section{Éléments pour la didactique : savoirs stabilisés}

\section{Thème/rhème vs sujet/prédicat}

L'opposition thème/rhème ne doit pas être confondue avec l'opposition sujet/prédicat, telle qu'elle a été à l'origine proposée par la logique grecque. Ce type d'analyse logico-philosophique vise en effet la connaissance des conditions de vérité d'un discours permettant d'accéder à l'essence des êtres et des choses, tandis que l'opposition thème/rhème, quand elle est présente dans un énoncé, est au contraire d'ordre non pas logique, mais informationnel et liée au discours : «elle permet de séparer dans un énoncé ce que l'on sait déjà (le thème) de ce qu'on apporte de nouveau (le rhème)» (Chiss, J.-L.; Filliolet, J. \& Maingueneau, M., 2001, p. 45). L'opposition thème/rhème a donné lieu à des analyses conduites tant au niveau de la phrase que du discours.

\section{Propriétés $d u$ thème et $d u$ rhème}

Contrairement aux rhèmes, les éléments thématiques d'un énoncé sont reconnaissables au fait qu'ils ne peuvent être ni interrogés, ni niés. Ainsi, dans la phrase Marie part en vacances en août, c'est en aô̂t ou partir en vacances qui sera rhématique selon que l'on aura respectivement (1), (2) ou (1'), (2') :

(1) Est-ce en août que Marie part en vacances?

(1') En août, Marie, est-ce qu'elle part en vacances?

(2) Ce n'est pas en août mais en septembre que Marie part en vacances.

(2') En août, ce n'est pas en vacances que Marie part, mais en stage.

\section{Signalisation du thème et $d u$ rhème en français contemporain}

La signalisation du thème et du rhème se manifeste différemment selon que l'on a affaire à une langue flexionnelle ou non. Dans une langue comme le français, où c'est en général l'ordre des mots qui supplée à la flexion, la signalisation du thème et du rhème se fait par le recours à des procédés spécifiques. 
Ainsi, le français emploie fréquemment des constructions à détachement qui permettent de distinguer le thème du sujet syntaxique, le thème détaché étant ensuite repris à l'intérieur de la phrase par un pronom clitique sujet: Cette voiture, elle roule vite. Ces détachements s'appliquent également à d'autres fonctions grammaticales : A Paris, j'y vais chaque semaine; ces restaurants, je les connais tous.

La réponse à une question est rhématique. Dans nombre de cas, on pourra trouver les mêmes éléments dans la question et la réponse. On recourra alors au test de l'affinité avec une position frontale, caractéristique du thème, ou au test de la construction clivée, caractéristique du rhème, pour distinguer le thématique du rhématique :

(4) Tuprends tes vacances quand?

- Mes vacances, je les prends au mois d'août.

???- Au mois d'août, mes vacances, je les prends.

(5) C'est quand que tu prends tes vacances?

* C'est tes vacances que tu prends quand?

A l'oral, le rhème est très souvent introduit par le marqueur c'est et ponctué en fin d'énoncé par hein ou par quoi, avec par ailleurs un contour intonatif en forme de cloche (bas-haut-bas, voir Danon-Boileau-Morel, 1998) :

(6) Les premières $A$, c'est des élèves plutôt littéraires hein.

Lorsque le sujet grammatical d'une phrase occupe une position rhématique, il n'est pas rare de trouver celui placé après le verbe, alors que le français est en principe une langue de type SVO dans l'énoncé déclaratif (voir Marchello-Nizia, 1999). On rencontre en particulier ce type de construction dans les énumérations ou lorsque certains adverbes ou compléments circonstanciels sont mis en tête de phrase :

(7) Vient ensuite un troisième élément.

(8) Au loin brillait une lumière.

On remarquera que la construction avec complément circonstanciel en tête de phrase accepte alors difficilement un complément direct

(9) * Au loin éclairait une lumière les jeunes gens (voir Blinkenberg, 1928, p. 104).

Dans d'autres cas, lorsque par exemple le rhème est l'agent d'un verbe, c'est alors une construction passive qui est tout naturellement préférée à un ordre SVO :

(10) On a découvert dans un champ 450 obus datant de la première guerre mondiale. Le premier obus a été déconvert par un agriculteur en train de labourer.

L'affinité, bien compréhensible en français, du thème pour les positions frontales, n'interdit cependant pas à un rhème de se trouver en tête de phrase: Excellent, ce fromage! Ces phénomènes sont en particulier fréquents à l'oral où les signaux prosodiques (contours intonatifs et accents d'intensités) permettent de thématiser ou de rhématiser n'importe quel constituant du discours, indépendamment de sa place : 
(11) Une année $\uparrow$ il a babité Paris $\downarrow$ (une année = rhème)

(12) Une année $\downarrow$ il a habité Paris $\downarrow$ (une année = thème)

D’après Sechehaye (1926, p. 33), cette autre ordonnance «correspond à quelque chose de plus subjectif dans le mouvement des idées. Celui qui parle exprime d'abord ce qu'il y a de dominant dans sa pensée, le prédicat de sa communication ». Notons enfin que le thème peut ne pas être exprimé du tout: Magnifique!, s'exclamera par exemple le visiteur de musée devant un tableau.

\section{Diachronie}

Semblable signalisation du thème et du rhème est caractéristique du français contemporain et liée au fait, qu'à partir du $15^{\mathrm{e}}$ siècle, l'ordre des mots est devenu fixe. Il en allait tout autrement en ancien français. Du 9e au 13e siècle, la langue comporte en effet encore deux cas (le cas-sujet et le cas-régime) et l'ordre des mots est beaucoup plus libre. L'ancien français est une langue où le verbe se met en deuxième position et où c'est toujours le thème de l'énoncé, quelle que soit sa fonction grammaticale dans la phrase, qui occupe la première place : Dis mules blanches fist amener Marsilie (La Chanson de Roland, cité par Marcello-Nizia 2003, p. 67). La syntaxe épouse donc la structure informationnelle de l'énoncé, ce qui ne sera plus possible lorsqu'à partir du $14^{\mathrm{e}}$ et $15^{\mathrm{e}}$ siècles, le sujet viendra systématiquement se placer devant le verbe et le complément d'objet direct derrière. Marcello-Nizia (2003) fait remarquer que la construction disloquée, fréquemment utilisée en français parlé contemporain pour signaler le thème, est très ancienne, puisqu'on la trouve déjà dans les textes littéraires du $11^{\mathrm{e}}$ siècle. Cette construction a cependant changé de valeur. Ce qu'elle indiquait au Moyen Âge dans l'usage littéraire, c'était en effet l'introduction d'un nouveau thème : Li niés Marsilie, il est venuz avant: " voici que s'avance le neveu de Marsilie »(Marcello-Nizia, 2003, p. 71), tandis que, d'après cet auteur, cette construction indique aujourd'hui, dans l'usage oral, la continuation du même thème (Pierre, je l'ai vu hier).

\section{Analyse textuelle}

Dans le domaine de l'analyse textuelle, l'opposition thème/rhème a été utilisée pour identifier trois types fondamentaux de progressions : la progression linéaire, la progression à thème constant et la progression à thèmes dérivés (voir Combettes 1983). Dans le premier cas, le rhème de la phrase précédente devient le thème de la phrase suivante : Pierre (Th1) a croisé Marie (Rh 1). Elle (Th2) sortait de chez, le coiffeur. Dans les progressions à thème constant, c'est toujours le même élément qui se trouve repris en position thématique : Le coucou a des habitudes différentes de celles des autres oiseaux. Il ne fait pas de nid et n'élève pas ses petits. Il confie ce soin à de petits passereaux. Enfin, ce qui caractérise la progression à thèmes dérivés, c'est la dérivation de thèmes différents à partir d'un hyperthème: La famille Dupont habite Paris. Gérard, le père, travaille à La Défense. Carole, la mère, est institutrice. Les enfants, Cédric et Maxence, sont étudiants à l'université. 


\section{Le fabuleux thème constant d'Amélie}

Quand la nuit tombat la petite fille entendu des hurlement. Alors elle se levat pour aller voir ce qui se passer et elle vu que la sorcière faisait un bouillont de sorcellerie. Avec une si petite voit, Amélie dit « mes pour qui est ce bouillont? »

«Mais ses pour toi mon enfant»

Amélie but la potion et elle fu transformer en bette sauvage.

(texte d'une élève de CE2 recueilli par C. Tisset, IUFM de Versailles)

L'utilisation d'une progression à thème constant est fréquente dans les récits d'enfants. L'est par contre beaucoup moins le fait que le maintien du thème soit assuré par une forme passive, ce qui prouve un très bon niveau langagier chez. cette élève. Les recherches effectuées dans le domaine de l'analyse textuelle sont donc particulièrement utiles dans le domaine de la didactique $d u$ français langue maternelle, car elles permettent d'évaluer la compétence écrite des élèves autrement que par le simple recours à l'orthographe.

Les chercheurs ont ensuite étudié de quelle façon les différents types de progression mentionnés supra se manifestent dans les grands modèles d'écriture (textes argumentatifs, narratifs, descriptifs). A l'opposition thème/rhème est alors venue s'ajouter la distinction cohésion/connexité (voir Bronckart, 1996). La cohésion désigne des faits de progression liés à des éléments d'ordre linguistique (anaphores, cataphores). La connexité ne concerne pas seulement le niveau de la réalisation linguistique, mais a aussi à voir avec l'interprétabilité des énoncés. Un énoncé textuellement bien formé exige absence de contradiction, progression, cohésion et connexité. Ainsi, une séquence comme Les Barbares sont aux portes de Rome, Marie fait la lessive est un énoncé qui ne présente ni progression, ni cohésion, ni connexité. Par contre dans Pierre a attrapé une maladie contagieuse, mais elle s'est déclarée hier, la cohésion est assurée, mais pas la connexité.

\section{Approcbe contrastive}

Dans les langues flexionnelles, la mise en position frontale suffit le plus souvent à indiquer la valeur thématique d'un constituant, comme le montre la paire de phrases suivantes empruntée au polonais (voir Adamzcewski, 1991, p. 211) :

(13) Szefem pánstwa zostat wybrany $X$.

(14) X zostat wybrany szefem pánstwa.

qui pourrait se gloser en français par :

(13') Sachant que quelqu'un a été élu chef de l'Etat, j'affirme que c'est X.

(14') Sachant que X a été élu quelque chose, j'affirme que c'est chef de l'Etat.

et se traduire par, en l'adaptant au contexte politique français :

(13”) Désormais le Président de la république, c'est X

(14") Désormais X est le Président de la république 
De même, dans une langue flexionnelle comme le russe, le recours à une construction passive, pourtant possible dans cette langue, n'est pas nécessaire pour traduire une phrase comme (7)

Pervyj snarjad obnarǔ̌il krest’janin, kotoryj paxal pole (littéralement en français : Le premier obus a découvert un paysan en train de labourer son champ).

Le complément d'objet direct le premier obus peut précéder le verbe à la forme active a découvert et se mettre en position frontale, tandis que le sujet syntaxique un agriculteur peut se placer derrière le verbe, sans que pour autant les fonctions grammaticales soient confondues. Le russe étant une langue à cas, le mot agriculteur aura en effet la forme krestjanin ou krestjjanina selon que l'on aura respectivement affaire au sujet grammatical ou au complément d'objet direct.

L'on ne s'étonnera donc pas que l'apprenant de langue étrangère, natif d'une langue à déclinaisons et donc peu rompu aux procédés de marquage de thème et du rhème spécifiques au français contemporain, ait tendance à transférer l'ordre des mots de sa langue maternelle. Ainsi, comme le signale Adamczewski (1991, p. 211), l'apprenant polonophone de français oublie fréquemment de reprendre par un pronom clitique un thème non sujet grammatical placé en position frontale: Les adresses j'ai trouvé dans l'annuaire vs Les adresses, je les ai trouvées dans l'annuaire.

\section{Questions ouvertes pour la recherche}

La dichotomie thème/rhème a suscité des recherches fécondes dans différents domaines : analyse du français parlé (voir Morel \& Danon-Boileau, 1998, et pour l'intonation Marandin, 2002), étude des phénomènes de grammaticalisation (voir Noailly 1982, Danon-Boileau \& Morel 1997) et linguistique de propriétés.

Ont entre autres retenu l'attention des chercheurs les phénomènes de dislocation droite, qualifiés selon les auteurs de « countertopic » (Lambrecht, 1981) ou de postrhème (Danon-Boileau \& Morel, 1997). Apparemment sortes de miroir inversé de la dislocation gauche, ceux-ci n'en partagent pourtant ni les propriétés syntaxiques (voir Fradin 1988), ni les traits intonatifs (voir Danon-Boileau \& Morel, 1998).

A la suite de Noailly (1982), Danon-Boileau \& Morel (1998) se sont intéressées aux noms prépositionnels comme question, point de vue, niveau et côté et ont montré que ceux-ci étaient des «introducteurs de cadrage thématique ». D'après ces deux auteurs, si l'objet sur lequel porte le discours est considéré comme n'étant pourvu d'aucune structure particulière, c'est question ou point de vue que le locuteur choisira. Si au contraire, l'objet sur lequel porte le discours est conçu comme un ensemble nettement structuré et organisé, c'est côté ou nivean qui sera utilisé. De fait, il a pu être établi que niveau était propre aux transactions dont le script présuppose un effet de liste : un hyperthème se déclinant en un ensemble de sous-thèmes indépendant les uns des autres (voir dans Flament-Boistrancourt, 2002, les scènes d'agence de voyage du corpus Lancom).

Dans le domaine de la linguistique de la phrase et des propriétés, la dichotomie thème/rhème a donné lieu à nombre d'analyses d'une grande finesse. Les recherches menées ont montré que les éléments susceptibles de se trouver en position rhématique 
ou thématique étaient de nature très diverse et ne se limitaient pas à des syntagmes nominaux ou verbaux. Ainsi Anscombre (1990a) et Nazarenko (2000) considèrent par exemple comme des formes de thèmes les subordonnées de cause introduites par puisque:

(15) (Puisque /*parce que) tu es si malin, essaie donc de résoudre ce problème.

(16) Pourquoi Pierre est-il absent?/(Parce qu'il/*Puisqu'il) est malade.

De même, un auteur comme Leeman 1987 montre que les expressions du type A ma grande surprise sont elles aussi des formes de thèmes, puisqu'elles en partagent nombre de propriétés.

On remarquera par ailleurs que l'opposition thème/rhème n'est pas forcément de l'ordre de constantes sémantiques. Ainsi, ce n'est pas parce que le couple parce que/puisque relève souvent d'une opposition de type thème/rhème, que la conjonction parce que ne peut pas ne pas être utilisée pour introduire une proposition à valeur thématique: On écoute l'instituteur parce qu'il a fait des études vs Parce qu'il a fait des études, on écoute l'instituteur (voir Nazarenko, 2000, p. 56).

Retiendront également l'attention d'un auteur comme Anscombre (1990b, c) les constructions du type donner ordre ou prêter attention. Cet auteur montre à leur propos que le $\mathrm{N}$ de ces constructions est précédé d'un article zéro, qui a pour fonction d'installer le $\mathrm{N}$ dans une position thématique (d'ouvrir, selon l'expression de cet auteur, " un espace discursif »). Les propriétés de ce type de construction sont en effet nettes : affinité avec la position frontale :

(17) A la nuit tombante, (ordre/l'ordre) fut donné aux soldats de se replier

(18) A la nuit tombante fut donné (*ordre/ l'ordre) aux soldats de se replier.

et impossibilité de nier la notion installée ou de la parcourir en allant dans le sens d'une polarité négative :

(19) Cette porte (donne/*interdit) accès à la bibliothèque

(20) Pierre avait toujours prêté (grande/* discrète) attention à la toilette féminine

On trouve également chez le même auteur (voir Anscombre, 1992) une analyse de l'opposition imparfait/composé présentée comme justiciable d'une dichotomie thème/rhème en ce qui concerne la distribution de la temporalité. L'imparfait présuppose en effet toujours un thème de nature temporelle :

(21) ??La France s'appelait la Gaule.

(22) Autrefois, la France s'appelait la Gaule.

tandis que, dans la phrase au passé composé, c'est dans le propos que l'information temporelle se trouve exprimée :

(23) Jean a-t-il vécu à Paris l'année dernière? Oui, mais six mois seulement

(24) ???Jean vivait-il à Paris l'année dernière? Oui, mais six mois seulement. 
De son côté, un auteur comme Lagae (1998), qui s'est intéressée aux constructions en $\mathrm{SN}+$ de+adj. montre que ce qui fait la spécificité de ces constructions et les oppose aux attributives (ily a un porto de versé vs un porto a été versé), c'est le fait que la construction en $\mathrm{SN}+$ de+adj. rhématise le $\mathrm{SN}$. On comprend mieux de cette façon le peu d'affinité de cette construction pour les positions frontales, les SN noms propres et les déterminants définis :

(25) *Un porto de versé lui ferait plaisir

(26) *Ily a Pierre de malade

(27) Ily a trois personnes de malades

(28) *Ily a la bouteille de vide

(29) Ily a une bouteille de vide

Par ailleurs, ce travail montre aussi, en s'appuyant sur une analyse de propriétés distinctives, que le prédicat des grammairiens et le rhème sont des notions qui ne se recouvrent pas forcément. Ainsi, dans des constructions apparemment apparentées comme je croyais sa fille malade et elle a sa fille qui est malade, le prédicat malade est dans le premier cas rhème et dans le second thème.

Enfin, c'est encore à une opposition de type thème/rhème qu'il convient de se référer pour éclairer des différences de sens fort subtiles entre connecteurs (voir par exemple Flament-Boistrancourt, 1999 pour une étude sur la façon dont ainsi et aussi disent la consécution) :

(30) Pierre est travailleur, (aussi/*ainsi) il a réussi

(31) Venez.plus tôt, (*aussi/ ainsi) nous aurons le temps de bavarder).

L'identification du thème et du rhème d'un discours est donc fort complexe et ne se ramène pas, loin s'en faut, à l'observation de structures de surface. 
L'entrée thème/rhème du glossaire de Comenius

\section{BIBLIOGRAPHIE}

\section{Fondamentaux}

BALLY, Ch., 1932, rééd. 1965, Linguistique générale et linguistique française, Berne, A. Francke.

Blanche-Benveniste, C., 1997, Approches de la langue parlée en français, Gap-Paris, Ophrys (Coll. L'essentiel français).

BRONCKART, J.-P., 1985, Le fonctionnement des discours: un modèle psychologique et une méthode d'analyse, Neuchâtel-Paris, Delachaux \& Niestlé éditeurs.

Bronckart, J.-P.,1996, Activité langagière, textes et discours. Pour un interactionisme socio-discursif, Lausanne, Delachaux \& Niestlé.

Cabier de praxématique, 30, 1998, «Les opérations de thématisation en français », Université Paul Valéry de Montpellier.

Chiss, J.-L., Filliolet, J. \& Maingueneau, D., 2001, Introduction à la linguistique française: syntaxe, communication et poétique, Paris, Hachette (Coll. Les fondamentaux, Hachette supérieur), tome 2, p. 67-68.

Combettes, B., 1983, Pour une grammaire textuelle : la progression thématique, Bruxelles-ParisGembloux, De Boeck-Duculot.

Dupont, D., Fauvaux, T. \& GHenet, M., 1994, La dynamique de linformation : éléments de grammaire textuelle, Bruxelles, De Boeck-Duculot (Coll. Formation continuée).

Galmiche, M., 1992, «Au carrefour des malentendus : le thème », L'information grammaticale, 54, Paris, Société pour l'Information grammaticale, p. 3-10.

L'information grammaticale, 54, 1992, "Le thème », Paris, S.I.G.

MARCELLO-NiziA, Chr., 1999, Le français en diachronie : douze siècles d'évolution, Paris-Gap, Editions Ophrys.

Neveu, F., 2000, Lexique des notions linguistiques, Paris, Nathan (Coll. 128)

Reichler-Beguelin, M.-J. et al., 1988, Ecrire en français : cohésion textuelle et apprentissage de l'expression écrite, Neuchâtel-Paris, Delachaux \& Niestlé (Coll. Techniques et méthodes pédagogiques).

Riegel, M., Pellat, J.-Chr. \& Rioul, R., 1994, Grammaire méthodique du français, Paris, Presses Universitaires de France (Coll. Linguistique nouvelle).

WeINRICH, H., 1989, Grammaire textuelle du français, Paris, Didier-Hatier. 


\section{Recherche}

Adamczewski, H., 1991, Le français déchiffré, Paris, Armand Colin.

Anscombre, J.-Cl., 1984, «La représentation de la notion de cause dans la langue », in Cabiers de grammaire de Toulouse, 8, Presses Universitaires du Mirail, p. 1-53.

ANSCOMBRE, J.-Cl., 1990a, "Thème, espaces discursifs et représentation évènementielle », in Fonctionnalisme et pragmatique, Anscombre, J.-Cl. \& Zaccaria, G. (eds), Milan, Ed. Unicopoli, p.43-150.

Anscombre, J.-Cl., 1990b, "Article zéro et structuration d'événements », in Charolles, M. \& Fichez, S. \& J. Jayez (éds.), Le discours, représentations et interprétations, Presses Universitaires de Nancy, p.265-305.

ANSCOMBRE, J.-Cl. (1990c) : «Espaces discursifs et contraintes adjectivales sur les groupes nominaux à article zéro », in De Mulder, W., Schuerewegen, F. \& L. Tasmowski (éds.), Enonciation et parti pris, Actes du colloque de l'Université d'Anvers, p.17-33.

Anscombre, J.-Cl., 1992, "Imparfait et passé composé : des forts en thème/propos », in L'information grammaticale, 55, p.43-53.

Blinkenberg, A., 1928, L'ordre des mots, Copenhague, Munskgaard.

Danon-Boileau, L. \& M.-A. Morel, 1997, "Question, point de vue, genre, style ...: les noms prépositionnels en français », in Faits de langues, 9, Gap-Paris, Ophrys, p. 193-200.

Flament-Boistrancourt, D., 1999, "Quelques aspects de ainsi et aussi consécutifs à la lumière d'un point de vue de non-francophone», in Le gré des langues, 15, Paris, L'Harmattan, p.142-179.

Flament-BOistranCOURT, 2002, "Et au niveau tarifs, ça donne quoi ? L'éclairage du corpus LANCOM sur le mot niveau», in Binon, J. \& Desmet, P. \& Elen, J. \& Mertens, P. \& L. Sercu (éds.), Feestbundel Mark Debrock: Tableaux vivants. Opstellen over taal-en-onderwijs aangeboden aan Mark Debrock, Leuven, Universitaire Pers Leuven, p. 117-132.

Fradin, B., 1988, «Approche des constructions à détachement : la reprise interne », in Langue française, 78, Paris, Larousse, p. 26-56.

Furukawa, N., 1996, Grammaire de la prédication seconde: formes, sens et contraintes, Louvain-laneuve, Duculot (Coll. Champs linguistiques).

GabelentZ, G. von der, 1891, rééd. 1984, Die Sprachwissenschaft : ihre Aufgaben, Methoden und bisherigen Ergebnisse, Tübingen, Gunter Narr.

Guimier, C. (éd.), 2000, La thématisation dans les langues, Actes du Colloque de Caen (9-11 octobre 1997), Peter Lang.

HALLIDAY, M.A.K., 1967, «Notes on transivity and theme in English », in JL3, p.37-82; 199244; JL4, p.179-215.

Hocketт, Ch., 1958, A Course in Modern Linguistics, New-York 
Langue française, 78, 1988, «Le thème en perspective », Paris, Larousse.

Lagae, V., 1998, Les constructions en «de» + adjectif. Typologie et analyse, Leuven, Presses universitaires de Louvain.

LAMBreCHT, K., 1981, Topic, antitopic and verb agreement in non-standart French, Amsterdam, John Benjamins B.V.

LEEman, D., 1987, "A ma grande surprise », in Revue québécoise de linguistique, 1/2, Montréal, Université du Québec, p.225-266.

Marandin, J.-M. et al., 2002, «Discourse making in French : C accents and discourse moves », in Bel, B \& Marlien, I. (eds.), Proceedings of the Speech Prosody 2002 Conference, Aix-enProvence (Laboratoire Parole et Langage), p. 471-474.

MARCELlo-Nizia, Chr., 2003, «Le français dans l'histoire », in Yaguello, M. (éd.), Le grand livre de la langue française, Paris, Editions du seuil, p. 11-90.

Marouzeau, J., 1938, L'ordre des mots dans la phrase latine. Le verbe, Paris, Belles Lettres.

Morel, M.-A. \& L. Danon-Boileau, 1998, Grammaire de l'intonation : l'exemple du français, GapParis, Editions Ophrys (Bibliothèque de Faits de langues).

NAZAREnKO, A., 2000, La cause et son expression en français, Gap-Paris, Ophrys (Coll. L'essentiel français).

NoAilly, M., 1982, "Côté, question et quelques autres », in Linguisticae Investigationes, VI/2, Amsterdam, John Benjamins B. V., p. 333-343.

NøLKE, H., 1994, Linguistique modulaire : de la forme au sens, Louvain-Paris, Éditions Peeters.

Paul, H., 1880, réed. 1975, Prinæipien des Sprachgeschichte, Tübingen, Max Niemayer Verlag.

SECHEHAYE, A., 1926, Essai sur la structure logique de la phrase, Paris, Champion.

SGALL, P., 1973, Topic, focus and generative semantics, Krönberg, Skriptor. 
